\title{
Optimal Planning of Nurses' Redeployment at Patient Overflow
}

\author{
Djamel Bellala, Lazhar Ourlis, Hacene Smadi \\ Control Engineering and Manufacturing Laboratory, University of Batna, 05000 Algeria \\ bellala_djamel@yahoo.co.uk, ourlisl@gmail.com,h.smadi@hotmail.fr
}

\begin{abstract}
In order to provide to his patients a quality medical service at lower cost, the General Administration Department of a University Hospital wants to maintain the number of the allocated nurses as low as possible while guaranteeing a satisfying level of health care. The nurses' redeployment is an optimization problem that falls under the category of integer linear programming problems whose graphical model is a digraph. The mathematical model is composed of an objective function of several interdependent variables to be obtained and some equality and side constraints that the General Administration Department should not violate in order to achieve satisfaction. The solution of this kind of problems rests on the use of an iterative method known as the simplex algorithm.
\end{abstract}

Keywords-medical care; nurses' redeployment; optimal planning; digraph; integer linear programming problem

\section{INTRODUCTION}

Hospital is considered as a service company which provides good medical care. Its prime importance is due to the fact of dealing with human being, but from a managerial point of view the hospital still a lucrative company which should be carefully managed because it is instantaneously exposed to an increase in the running costs. For this reason it falls to the manager to instantaneously optimize the working conditions of the hospital by namely providing an optimal planning of the overall tasks. The nurses' allocation during patient overflow is one of the tasks that the General Administration Department should optimize in order to accomplish its duty by guaranteeing a satisfying level of health care. At the time of patient overflow some services become saturated and the need for other free beds belonging to other services is of a vital character. Consequently the nurses' redeployment becomes more than necessary, therefore the nurses of the other services with fewer occupied beds are asked to join the other services with full occupied beds. So how can the General Administration Department perform this exercise in an objective manner? The teaching hospital of Batna, Algeria is the place where this study is carried out [1]-[2].

\section{NETWORK MODEL}

The network model is a directed graph (digraph) composed of a finite set of nodes and weighted arcs, say eight nodes (vertices) and sixteen weighted arcs (edges) see figure 1.

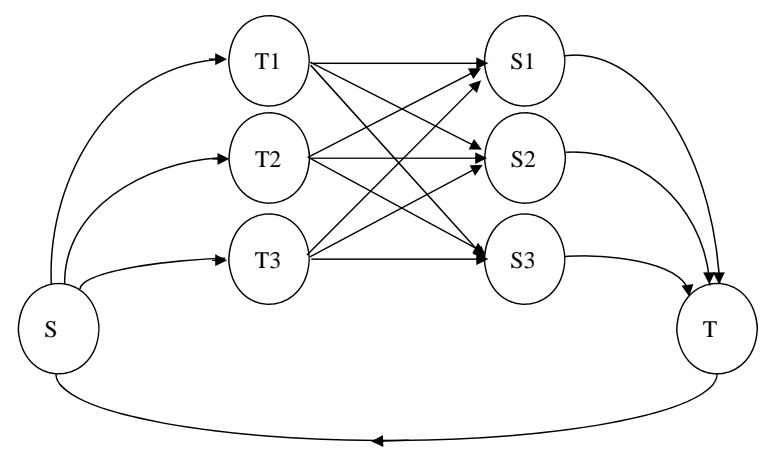

Figure 1. Network representation of nurses' redeployment

With:

node $\mathrm{S}$ : representing the source of nurses provided by the General Administration Department node $\mathrm{T}_{1}$ : represents the first team node $\mathrm{T}_{2}$ : represents the second team node $\mathrm{T}_{3}$ : represents the third team node $S_{1}$ : represents the first service node $S_{2}$ : represents the second service node $\mathrm{S}_{3}$ : represents the third service

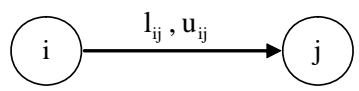

Figure 2. Typical representation of a weighted arc

The arcs which designate the connectivities between each pair of nodes are weighted with two different values; a lower one in the left and an upper one in the right as it is represented in figure 2. These lower and upper values represent the constraints of the problem such that the arcs entering nodes $T_{i}$ represent the source constraints with lower values representing the minimum numbers of necessary nurses that the General Administration Department should allocate to each team, whereas the upper values are the maximum ones. These upper values have to be obtained that is why they are not previously known. They are labeled as infinity. 
TABLE I. SOURCE CONSTRAINTS

\begin{tabular}{|c|c|c|r|r|r|r|}
\hline & \multicolumn{6}{|c|}{ Team } \\
\cline { 2 - 7 } & \multicolumn{2}{|c|}{$\mathrm{T}_{1}$} & \multicolumn{2}{|c|}{$\mathrm{T}_{2}$} & \multicolumn{2}{|c|}{$\mathrm{T}_{3}$} \\
\hline G. & $\mathrm{l}$ & $\mathrm{u}$ & $\mathrm{l}$ & $\mathrm{u}$ & $\mathrm{l}$ & $\mathrm{u}$ \\
$\mathrm{S}$ & $\mathrm{b}$ & $\mathrm{b}$ & $\mathrm{b}$ & $\mathrm{b}$ & $\mathrm{b}$ & $\mathrm{b}$ \\
\cline { 2 - 8 } & 3 & $\mathrm{I}$ & 3 & $\mathrm{I}$ & 2 & $\mathrm{I}$ \\
& 2 & nf. & 0 & nf. & 5 & nf. \\
\hline
\end{tabular}

Arcs going from a given node $T_{i}$ and entering a given node $S_{i}$ represent the team constraints where every two lower and upper values correspond to the range limit of nurses that every team can provide to every service. In a matrix form these constraints are given as follows:

$$
\left[\begin{array}{lll}
8-10 & 13-14 & 9-14 \\
6-8 & 13-14 & 9-14 \\
4-6 & 12-14 & 7-9
\end{array}\right]
$$

Figure 3. Limit values Team-Service

Where

Rows 1, 2 and 3 represent teams 1, 2 and 3 respectively

Columns 1, 2 and 3 represent services 1,2 and 3 respectively

Arcs entering node $\mathrm{T}$ represent the hospital constraints where the lower values represent the minimum number of nurses that the General Administration Department should allocate to every service in order to guarantee the reduced service only.

Numerical values of these constraints are represented in the following table.

TABLE II. SERVICES' CONSTRAINTS

\begin{tabular}{|c|c|c|r|c|c|c|}
\hline & \multicolumn{6}{|c|}{ Service } \\
\cline { 2 - 7 } & \multicolumn{2}{|c|}{$\mathrm{S}_{1}$} & \multicolumn{2}{|c|}{$\mathrm{S}_{2}$} & \multicolumn{2}{|c|}{$\mathrm{S}_{3}$} \\
\hline G. & $\mathrm{l}$ & $\mathrm{u}$ & $\mathrm{l}$ & $\mathrm{u}$ & $\mathrm{l}$ & $\mathrm{u}$ \\
$\mathrm{S}$ & $\mathrm{b}$ & $\mathrm{b}$ & $\mathrm{b}$ & $\mathrm{b}$ & $\mathrm{b}$ & $\mathrm{b}$ \\
\cline { 2 - 8 } & 1 & $\mathrm{I}$ & 3 & $\mathrm{I}$ & 2 & $\mathrm{I}$ \\
& 5 & nf. & 5 & nf. & 5 & nf. \\
\hline
\end{tabular}

\section{MATHEMATICAL MODEL}

The major concern of the General Administration Department is to distribute the minimum amount of nurses over the teams and services such that the medical care provided is at least acceptable or better. From Fig. 1, we remark that the number of nurses in question corresponds to the flow in the TS arc [3]-[5].

Since $x_{T S}$ represent the number of nurses flowing back to the source in order to be redistributed next time, we affirm that $x_{T S}$ is the number of nurses to be minimized. So the problem described as a minimum cost flowing problem and the objective function is given as follows:

Declaring that $x_{T S}$ is the number of nurses flowing back to the source, then the objective function is as follows:

$$
\text { Minimize } z=x_{T S}
$$

Since $x_{T S}$ can be determined in terms of all arc quantities, it's all the same if we calculate the optimal values of the $x$ vector which is composed of sixteen variables such that:

$$
x=\left(\begin{array}{l}
x_{S T_{1}}, x_{S T_{2}}, x_{S T_{3}}, x_{T_{1} S_{1}}, x_{T_{1} S_{2}}, x_{T_{1} S_{3}}, x_{T_{2} S_{1}}, x_{T_{2} S_{2}} \\
x_{T_{2} S_{3}}, x_{T_{3} S_{1}}, x_{T_{3} S_{2}}, x_{T_{3} S_{3}}, x_{S_{1} T}, x_{S_{2} T}, x_{S_{3} T}, x_{T S}
\end{array}\right)
$$

Subject to

Equality constraints:

$$
\begin{aligned}
& x_{S T_{1}}+x_{S T_{2}}+x_{S T_{3}}=x_{T S} \\
& \left\{\begin{array}{l}
x_{T_{1} S_{1}}+x_{T_{1} S_{2}}+x_{T_{1} S_{3}}=x_{S T_{1}} \\
x_{T_{2} S_{1}}+x_{T_{2} S_{2}}+x_{T_{2} S_{3}}=x_{S T_{2}} \\
x_{T_{3} S_{1}}+x_{T_{3} S_{2}}+x_{T_{3} S_{3}}=x_{S T_{3}}
\end{array}\right. \\
& \left\{\begin{array}{l}
x_{T_{1} S_{1}}+x_{T_{2} S_{1}}+x_{T_{3} S_{1}}=x_{S_{1} T} \\
x_{T_{1} S_{2}}+x_{T_{2} S_{2}}+x_{T_{3} S_{2}}=x_{S_{2} T} \\
x_{T_{1} S_{3}}+x_{T_{2} S_{3}}+x_{T_{3} S_{3}}=x_{S_{3} T}
\end{array}\right. \\
& x_{S_{1} T}+x_{S_{2} T}+x_{S_{3} T}=x_{T S}
\end{aligned}
$$

Side constraints

$$
\left\{\begin{array}{l}
x_{S T_{1}} \geq 32 \\
x_{S T_{2}} \geq 30 \\
x_{S T_{3}} \geq 25
\end{array}\right.
$$

$$
\begin{aligned}
& \left\{\begin{array}{l}
8 \leq x_{T_{1} S_{1}} \leq 10 \\
13 \leq x_{T_{1} S_{2}} \leq 14 \\
9 \leq x_{T_{1} S_{3}} \leq 14 \\
6 \leq x_{T_{2} S_{1}} \leq 8 \\
13 \leq x_{T_{2} S_{2}} \leq 14 \\
9 \leq x_{T_{2} S_{3}} \leq 14 \\
4 \leq x_{T_{3} S_{1}} \leq 6 \\
12 \leq x_{T_{3} S_{2}} \leq 14 \\
7 \leq x_{T_{3} S_{3}} \leq 9
\end{array}\right. \\
& \left\{\begin{array}{l}
x_{S_{1} T} \geq 15 \\
x_{S_{2} T} \geq 35 \\
x_{S_{3} T} \geq 25
\end{array}\right. \\
& x_{T S} \geq 0
\end{aligned}
$$

The obtained model is called a linear programming model with an objective function to be minimized (equation 1 ) and some constraints. Equations 2 up to 5 represent the vertex conservation, i.e. what enters a node is the same as 
what goes out, which shows that all these equations are node constraints. The rest of the equations 6 up to 9 are describing the constraints related to capacities, i.e. the allocation ranges related to both services and teams, we thus speak about arc constraints.

\section{PROBLEM-SOLVING ALGORITHM}

Based on the obtained mathematical model we are asked to find an optimal solution that satisfies all the constraints, i.e. the solution that yields the best (minimum in our case) value of the objective function.

The most popular algorithm for solving this type of linear programming problems is the so called simplex algorithm, which is made up for three basic stages [3]:

- Initialization: the algorithm starts with a basic feasible solution provided that the right hand side of every constraint is always non negative.

- Iterations: the algorithm goes through intermediate iterations where solution with better values of the objective function are found.

- Termination: the algorithm terminates where there is no entering variable to provide optimal solution.

In order to solve numerically this problem, the famous fourth-generation programming language, Matlab system is utilized. The latter is composed of several optimization toolboxes which provide useful algorithms to solve quite a lot of engineering problems. In our case the choice is made upon the linprog function whose arguments are listed below [6]-[8].

$x=\operatorname{linprog}\left(f, A, b, A_{e q}, b_{e q}, l_{b}, u_{b}, x_{0}\right.$, options $)$

with :

$f \quad$ Linear objective function vector

A Matrix for linear inequality constraints

$b \quad$ Vector for linear inequality constraints

$A_{e q} \quad$ Matrix for linear equality constraints

$b_{e q} \quad$ Vector for linear equality constraints

$l_{b} \quad$ Vector of lower bounds

$u_{b} \quad$ Vector of upper bounds

$x_{0} \quad$ Initial point for $\mathrm{x}$

Since we have no inequality constraints in our model, we set matrix $A$ and vector $b$ as empty ones according to the linprog statements. So,

$$
\begin{aligned}
& A=[] \\
& b=[]
\end{aligned}
$$

$A_{e q}$ is the incidence matrix whose elements are determined from the weighted graph of figure 1 :

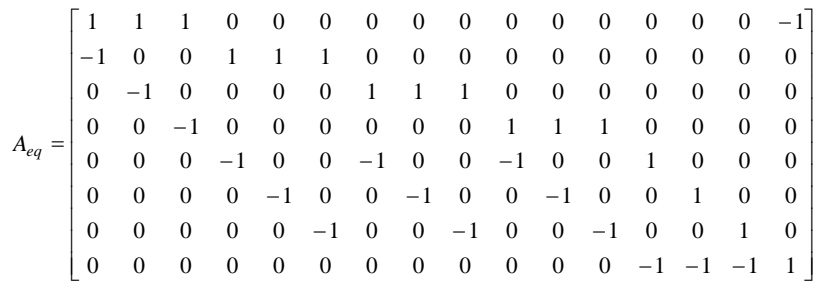

Figure 4. Incidence matrix

The initial value vector $x_{0}$ of the design vector is chosen to be a null vector.
Since the number of redeployed nurses to be obtained is a real world quantity, so only whole numbers are acceptable, then the simplex algorithm to be utilized should take into account the fact that the result should be given with integer numbers only. For this reason the options feature of the linprog function should be set as follows:

options = optimset('LargeScale', 'off', 'Simplex', 'on')

By adding this option to the algorithm, the problem becomes an integer linear programming problem.

$b_{e q}, u_{b}$, and $l_{b}$ express the right hand side of the equality constraints, the upper values and the lower values vectors respectively.

Note that the letter $M$ denotes the infinity value chosen to be equal to 1000 within Matlab system.

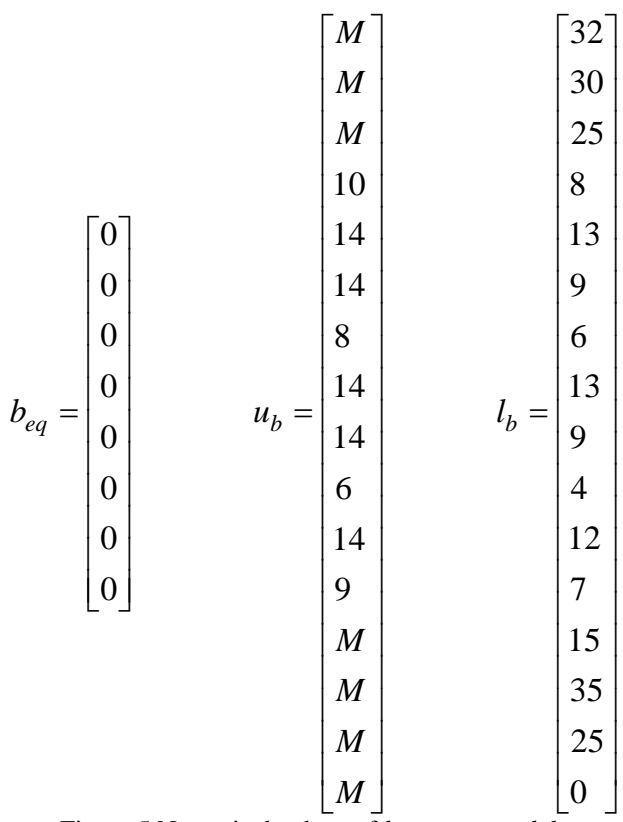

Figure 5.Numerical values of $b_{e q}, u_{b}$, and $l_{b}$

\section{RESULTS AND DISCUSSION}

After entering accurately the data into a Matlab file, we have carried out correctly the execution of the program. Notice that the algorithm is deterministic and the obtained result if there is any is an exact one. The programme converges to an optimal vector of sixteen interdependent variables which are all integers. Numerical values of the design variables of the $x$ vector are given below:

$$
x=\left(\begin{array}{l}
x_{S T_{1}}=32 ; x_{S T_{2}}=30 ; x_{S T_{3}}=25 ; \\
x_{T_{1} S_{1}}=10 ; x_{T_{1} S_{2}}=13 ; x_{T_{1} S_{3}}=9 ; \\
x_{T_{2} S_{1}}=8 ; x_{T_{2} S_{2}}=13 ; x_{T_{2} S_{3}}=9 ; \\
x_{T_{3} S_{1}}=6 ; x_{T_{3} S_{2}}=12 ; x_{T_{3} S_{3}}=7 ; \\
x_{S_{1} T}=24 ; x_{S_{2} T}=38 ; x_{S_{3} T}=25 ; \\
x_{T S}=87
\end{array}\right)
$$




\section{CONCLUSION}

This research paper allowed us to perform many interesting tasks, starting with the transformation of the problem from a descriptive model to both a network and mathematical ones. Other models can be developed; it depends only on mathematical and graphical skills of the researcher.

The obtained mathematical model is not only a linear programming model but whole values of the design variables are necessary because of the fact that they represent human being, in this case the nurses. This is why the use of integer linear programming algorithm.

The obtained results are in perfect harmony because the amount of nurses allocated to each team sum to 87, which in its turn corresponds to the same number as the one summing the nurses returning back to the General Administration Department. Also the number of nurses provided by each team to each service is the same as each team number. Consequently the node conservation is absolutely respected.

\section{REFERENCES}

[1] K. L. Teow, Practical Operations Research Applications for Healthcare Managers, Annals Academy of Medicine. Singapore, , 1 MSc (Industrial \& Systems Engineering) June 2009, Vol. 38 No. 6

[2] M. Benzouai and all, Towards a better control of the infectious risks associated with health care activities, International Journal of Computer Science Issues, 31st July 2013.

[3] G. Srinivasan, "Operations Research: Principles and Applications,”, PHI Learning Private Limited, second edition (C) 2010, New Delhi.

[4] H. A. Taha, "Operations Research an Introduction,”, Pearson International eighth edition 2007.

[5] F. S. Hillier G. J. Lieberman, "Introduction to Operations Research,”, McGraw-Hill international, ninth edition 2010.

[6] Singiresu S. Rao, "Engineering Optimization: Theory and Practice," Published by John Wiley \& Sons, Inc., Hoboken, New Jersey, Copyright (C) 2009.

[7] G. Recktenwald, "Numerical Methods with Matlab Implementation and Application," Prentice Hall, Upper Saddle River, New Jersey 07458, 2000.

[8] G. Lindfield, J. Penny, "Numerical Methods using Matlab," Prentice Hall, Upper Saddle River, New Jersey 07458, 2000. 